\title{
The Proteomic Expression of Nuclear Apoptosis-Inducing Factor1 (NAIF1) in Colorectal Tissues
}

\author{
Ahmed Arnaoty ${ }^{1}$, Yves Bigot ${ }^{2}$, Thierry Lecomte ${ }^{3}$ \\ ${ }^{1}$ Assistant Professor at Medenat AL-Elem University College Department of Nursing/Baghdad/Iraq, ${ }^{2}$ Director \\ of research at UMR INRA-CNRS 7247, INRA Val de Loire-France, ${ }^{3}$ Professor at Tours university, Department of \\ Hepato-Gastro-Enterology; Tours/France
}

\begin{abstract}
The neogenic recombinases are potentially a source of genetic variability possibly implicated in the mechanisms of genetic instability involved in the processes of carcinogenesis. One of these neogenic recombinases is the nuclear apoptosis-inducing factor 1(NAIF1), which codes the protein NAIF1 that induces apoptosis in various human cancers.

The aim of this work is to study the expression of NAIF1 neogene in cancerous and non-cancerous colorectal tissues.

The protein expression of NAIF1gene has been studied by western blot method in the samples of protein extracted from 29 patients with colorectal cancer.

The result of this study showed that the protein expression of NAIF1 was found in both cancerous and non-cancerous colorectal tissues, but it is highly expressed in non-cancerous tissues adjacent to cancerous one. Its expression is higher in non-metastatic cancerous tissues compared with metastatic one. Also this expression is higher in microsatellite instable (MSI) group of cancer compared with microsatellite stable (MSS) group.

It can be concluded that NAIF1 protein expression inversely related with more advanced stage or grade of colorectal cancer and it may have a role in inhibition of proliferation, migration and invasion of colorectal cancer by inducing apoptosis.
\end{abstract}

Keywords: DNA transposons, Domestication, Neogene, NAIF1, microsatellite instable, microsatellite stable, colorectal cancer.

\section{Introduction}

Colorectal cancers are solid tumors whose somatic genetic and epigenetic alterations are best documented ${ }^{[1]}$. They are relatively homogeneous tumors in terms of their anatomopathological characteristics as they are in more than $90 \%$ of cases of adenocarcinomas ${ }^{[1]}$. Two main groups of colorectal cancers characterized by different

\section{Corresponding Author:}

Ahmed Arnaoty

Assistant Professor at Medenat AL-Elem University

College Department of Nursing/Baghdad/Iraq

e-mail: ahmedarnaoty@yahoo.com somatic genetic alterations were presents ${ }^{[1-3]}$. The most frequent group is characterized by a chromosomal instability resulting in the recurrent loss of chromosomal segments $^{[4,5]}$, called LOH for Loss of heterozygoty and represents $85 \%$ of colorectal adenocarcinomas ${ }^{[2]}$. The other colorectal cancer group is characterized by microsatellite instability (MSI) due to a mismatch repair of DNA (MMR repair system) ${ }^{[2]}$.

However, a phenomenon called molecular domestication has been reported for some DNA transposons resulting in the formation of neogenes ${ }^{[6]}$. Most of these neogenes domesticated from DNA transposon encode proteins for the most part still poorly characterized and whose biological functions are poorly 
known. These proteins are involved in various biological processes that contribute directly or indirectly to genome stability (cell proliferation, cell cycle progression, chromatin modification, transcription...) ${ }^{[6,7]}$. Analysis of the human genome made it possible to identify 47 genes derived from transposable elements among which 43 genes are derived from domesticated DNA transposons ${ }^{[8]}$. One of these neogenes, which is selected in this study, is the neogenic recombinase nuclear apoptosis-inducing factor 1(NAIF1) code the protein NAIF1 that derived from the DNA transposon by molecular domestication. NAIF1 is a nuclear protein that contains a Myb-like domain at its $\mathrm{N}$-terminal region ${ }^{[9]}$.

The human gene encoding nuclear apoptosis inducing factor 1 (NAIF1) is located on chromosome $9 \mathrm{q} 34.11$, and reported to repress the progression of several human cancers ${ }^{[9,10-14]}$.

In gastric cancer ${ }^{[10,12]}$, prostate carcinoma ${ }^{[11]}$, in osteosarcoma cells $^{[13]}$, in non-small-cell lung cancer ${ }^{[14]}$. NAIF1 inhibit the progression of cancer by inducing apoptosis and it plays an important role in control of the expression of some pivotal anti-cancer or apoptosisrelated genes in physiological level ${ }^{[10,12]}$. These findings may indicate that it is very likely that NAIF1 may predominantly act as a tumor-suppressive gene, possibly through the activation of apoptotic pathways, in various types of cancers ${ }^{[10-14]}$. In the present study the model retained for the study of the expression of NAIF1 neogenic protein by the western blot method was an in vitro model of human colorectal cancerous and non-cancerous tissues, using the protein extracted from these tissues and by the antibodies synthesized by Arnaoty et $\mathrm{al}^{[15]}$, that allow the study of the expression and the analysis of neogenic recombinase corresponding to NAIF1 protein.

The aim of this study is to show the protein expression of NAIF1 in these colorectal tissues.

\section{Materials and Method}

The tumor samples and their Characteristics: Frozen samples of colorectal tumors (tumor tissue and adjacent non-tumoral colonic tissue) from 29 patients operated with colorectal cancer in 2007 or 2008 and stored in a tumor bank were selected for this study. The main characteristics of the included colorectal tumors are summarized in Table 1.

Table 1 : The main characteristics of the included colorectal tumoral and non-tumoral samples.

\begin{tabular}{|c|c|c|c|c|c|c|c|c|}
\hline $\operatorname{Pt} \mathbf{N}^{\circ}$ & Sex & $\begin{array}{l}\text { Tumor } \\
\text { cells } \%\end{array}$ & $\begin{array}{c}\text { Tumor } \\
\text { localization }\end{array}$ & Stage & Differentiation & Status MSI, MSS & BRAF & KRAS \\
\hline 1 & $\mathrm{~F}$ & $80 \%$ & Rt colon & 2 & Intermediate & MSI-H & Non mutated & \\
\hline 2 & M & $90 \%$ & Rt colon & 2 & Poor & MSS & & Non mutated \\
\hline 3 & M & $90 \%$ & Lt colon & 4 & Poor & MSS & & Non mutated \\
\hline 4 & $\mathrm{~F}$ & $90 \%$ & Lt colon & 2 & Intermediate & MSS & & G13D \\
\hline 5 & M & $90 \%$ & Sigmoid & 2 & Intermediate & MSS & & G12D \\
\hline 6 & M & $90 \%$ & Rt colon & 4 & Good & MSS & & G12A \\
\hline 7 & M & $90 \%$ & Rt colon & 3 & Poor & MSI-H & Non mutated & Non mutated \\
\hline 8 & M & $75 \%$ & Rt colon & 3 & Intermediate & MSS & & Non mutated \\
\hline 9 & $\mathrm{~F}$ & $80 \%$ & Rt colon & 3 & Poor & MSI-H & V600E & Non mutated \\
\hline 10 & $\mathrm{M}$ & $75 \%$ & Rt colon & 3 & Poor & MSS & & $\mathrm{G} 12 \mathrm{C}$ \\
\hline 11 & $\mathrm{M}$ & $90 \%$ & Rt colon & 1 & Intermediate & MSS & & G12D \\
\hline 12 & $\mathrm{M}$ & $90 \%$ & Rectum & 3 & Intermediate & MSS & & G13D \\
\hline 13 & $\mathrm{~F}$ & $50 \%$ & Rt colon & 3 & Intermediate & MSI & Non mutated & Non mutated \\
\hline 14 & $\mathrm{~F}$ & $80 \%$ & Rt colon & 3 & Poor & MSI-H & Mutated & \\
\hline 15 & $\mathrm{M}$ & $70 \%$ & Rt colon & 2 & Intermediate & MSI-H & V600E & \\
\hline 16 & $\mathrm{~F}$ & $50 \%$ & Rt colon & 2 & Intermediate & MSS & & G12S \\
\hline 17 & $\mathrm{M}$ & $50 \%$ & Sigmoid & 2 & Intermediate & MSS & & Non mutated \\
\hline
\end{tabular}




\begin{tabular}{|c|c|c|c|c|c|c|c|c|}
\hline $\operatorname{Pt} \mathrm{N}^{\circ}$ & Sex & $\begin{array}{l}\text { Tumor } \\
\text { cells \% }\end{array}$ & $\begin{array}{c}\text { Tumor } \\
\text { localization }\end{array}$ & Stage & Differentiation & Status MSI, MSS & BRAF & KRAS \\
\hline 18 & $\mathrm{~F}$ & $60 \%$ & Sigmoid & 2 & Intermediate & MSS & & Non mutated \\
\hline 19 & M & $50 \%$ & Lt colon & 2 & Intermediate & MSS & & Non mutated \\
\hline 20 & M & $50 \%$ & Lt colon & 2 & Intermediate & MSS & & Non mutated \\
\hline 21 & M & $55 \%$ & Sigmoid & 3 & Intermediate & MSS & & Non mutated \\
\hline 22 & $\mathrm{~F}$ & $50 \%$ & Rt colon & 3 & Good & MSS & & G12A \\
\hline 23 & $\mathrm{~F}$ & $65 \%$ & Lt colon & 3 & Poor & MSS & & Non mutated \\
\hline 24 & $\mathrm{~F}$ & $65 \%$ & Lt colon & 3 & Intermediate & MSS & & G12D \\
\hline 25 & $\mathrm{~F}$ & $65 \%$ & Rt colon & 3 & Poor & MSS & & Non mutated \\
\hline 26 & $\mathrm{~F}$ & $70 \%$ & Rt colon & 4 & Poor & MSI-H & Non mutated & Non mutated \\
\hline 27 & M & $80 \%$ & Lt colon & 4 & Poor & MSS & & G12D \\
\hline 28 & $\mathrm{M}$ & $50 \%$ & Lt colon & 4 & Intermediate & MSS & & Non mutated \\
\hline 29 & $\mathrm{~F}$ & $70 \%$ & Lt colon & 4 & Poor & MSS & & G12V \\
\hline
\end{tabular}

M: Male, F :Female, Rt :Right, Lt :Left, MSI : Microsatellite instable, MSI-H : Microsatellite instable high, MSS: Microsatellite stable.

Protein extractions from tumoral and nontumoral samples: Sections with a thickness of $10 \mu \mathrm{m}$ of the samples of tumor and non-tumor tissues preserved at $-80^{\circ} \mathrm{C}$, were made by using a cryostat in the department of histopathology/Trousseau hospital/Tours/France. From these tissue sections, protein lysates were prepared using an ice-cold RIPA buffer $(20 \mathrm{mM}$ Tris- $\mathrm{HCl}(\mathrm{pH}$ 7.2), $150 \mathrm{mM} \mathrm{NaCl}, 1 \mathrm{mM}$ EDTA, $10 \%$ glycerol, $1 \%$ Triton X- 100, 0.5\% deoxycholate, $0.1 \%$ SDS, $1 \mathrm{mM}$ DTT, 16 complete protease cocktail (Roche)). The protein extracts were assayed by the Bradford method and preserved at $-20{ }^{\circ} \mathrm{C}$.

Analysis of proteins by acrylamide gel electrophoresis: The electrophoresis technique under denaturing conditions SDS-PAGE was used. The total protein extracts were mixed with lysis buffer $(20 \%$ SDS, $100 \mathrm{mM} \mathrm{NaCl}, 10 \mathrm{mM}$ beta-mercaptoethanol, cocktail of protease inhibitors (Hoche Diagnostics $\mathrm{GmbH}$, Mannheim, Germany)), and then denatured for $5 \mathrm{~min}$ at $95^{\circ} \mathrm{C}$.

Fifty micrograms of protein extracts from cancerous and non-cancerous tissues were deposited in each well of a polyacrylamide gel.

Western Blot: After electrophoresis, the proteins were transferred to a polyvinylidene difluoride (PVDF) membrane (Bio-Rad, Richmond, USA) using "TransBlot ${ }^{\circledR}$ SD Semi-DryTransfer Cell” (BIO-RAD) in transfer buffer (40 mM Tris-base, 0.1M Glycine, 20\% ethanol).
The membranes were then incubated with the antiNAIF1 primary antibody (In Cell Art, Nantes/France) at a dilution of 1: 250 for two hours at room temperature. After three successive 10-minute washes then incubated with a secondary antibody coupled to Goat anti-Mouse IgG-HRP (Amersham, GE Healthcare) for 1 hour at room temperature. After three successive 10-minute washes the membranes were analyzed by means of a chemiluminescence reaction (Amersham ECL Advance Western Blotting Detection Kit, GE Healthcare) the images were collected using the FUGI LAS4000 imager. The membranes were then dehybridized and then rehybridized with a polyclonal anti-chicken actin antibody (Abcam, USA).

Analysis of the data obtained by western blot: For each patient, expression of the NAIF1 protein in cancerous colorectal tissue was compared to that observed in adjacent non-cancerous tissue. The expression results were then analysed according to different clinical and molecular parameters. The results were calculated by taking into account on the migration gels the band corresponding to the $35-\mathrm{kDa}$ isoform of NAIF1 expression. The Western blot signals were analysed with the Multi Gauge software (which determines the optical density of the bands obtained in Western Blot and corresponding to the protein expression and their contents of housekeeping protein, actin).

Statistical Analysis: We have performed statistical analyses to compare the two types of samples (cancerous 
(T) and non-cancerous ( $\mathrm{N})$ ), this is an adjustment of protein expression parameters as a function of stromal cell percentage over sample $\mathrm{T}$ or $\mathrm{N}$ for each selected patient. The Kolmogorov-Smirnov and Shapiro-Wilk test were used to test a difference in NAIF1 expression in these two types of samples [non-cancerous (N) versus cancerous $(\mathrm{T})]$, and for cancerous samples $(\mathrm{T})$ differentiate between non-metastatic/metastatic; MSI/ MSS; mutated KRAS, BRAF/non-mutated, A result is considered significant if $\mathrm{p} \leq 0.05$.

\section{Results}

Expression of the NAIF1 protein in colorectal tissues (cancerous \& non-cancerous): Results highlighted a unique product of expression of NAIF1 in all samples of colorectal tissues cancerous ( $\mathrm{T}$ ) and non-cancerous $(\mathrm{N})$ by western blot, corresponding to $35 \mathrm{kDa}$ molecular weight ${ }^{[17]}$ equal to that of the NAIF 1 transposase (figure 1,2) using the anti-NAIF1 antibody (In Cell Art, Nantes/France).

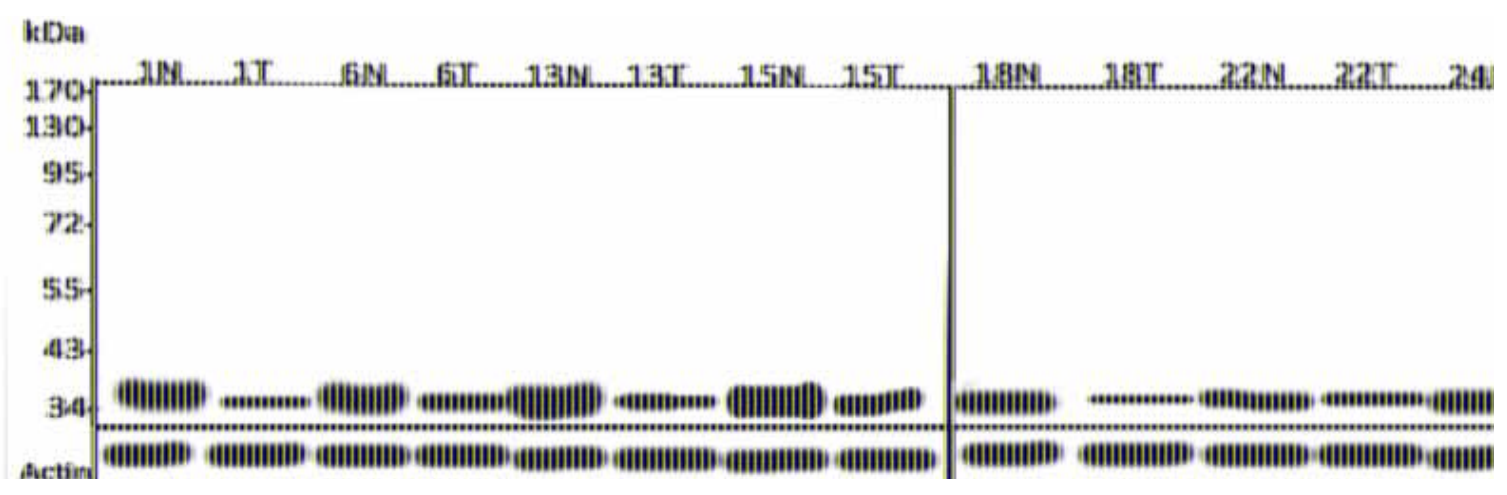

Figure 1: NAIF1 expression by western blot for 8 patients with antisera anti NAIF1. Lanes 1 to 16 correspond to protein extracts colorectal tissues $(\mathrm{N}, \mathrm{T})$ in 8 patients respectively. Hybridizing the membranes with a specific monoclonal antibody actin, in each lane. Molecular weights are indicated in the left margins. Molecular weight of NAIF1 is indicated in the right margin.

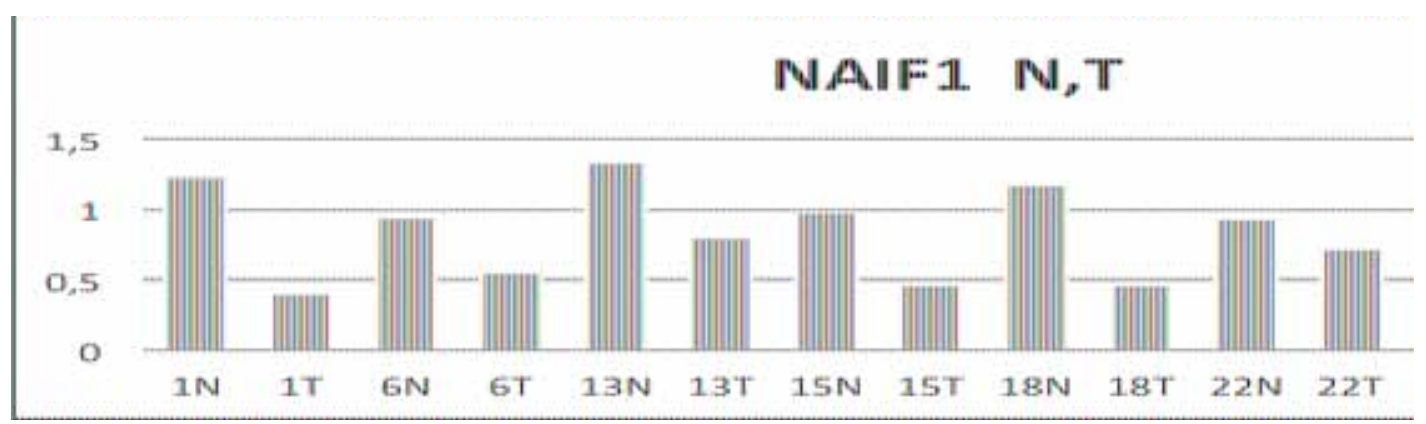

Figure 2: NAIF1 expression by western blot represented by Histogram for protein extracts of 8 samples with anti NAIF1.

The protein expression of the NAIF1 gene was analysed according to different parameters figures 3 :

We compared the expression of NAIF1 between the two types of samples non-cancerous tissues $(\mathrm{N})$ versus cancerous tissues $(\mathrm{T})$ by adjusting them according to the percentage of stromal cell on the $\mathrm{N}$ and $\mathrm{T}$ sample. This comparison showed that the level of expression of NAIF1 in $\mathrm{N}$ is significantly higher than that observed in $\mathrm{T}(\mathrm{p}$-value $=0.0001)$.

Also we compared the expression of NAIF1 for the cancerous samples (T) according to the status of phenotype microsatellite instable (MSI) or microsatellite stable (MSS).

We observed that NAIF1 expression of T in MSI tumors was significantly greater than that observed in MSS tumors ( $p$-value $=0.0001)$.

Then we compared the expression of NAIF1 for cancerous samples $(\mathrm{T})$ according to the metastatic or non-metastatic stage of cancer. There is a significant difference observed in NAIF1 expression in the metastatic (low expression) versus non-metastatic (high expression) stage of cancer, high expression in non- 
metastatic compared with metastatic one (p-value = 0.0001).

Finally, we compared the expression of NAIF1 for cancerous samples ( $\mathrm{T}$ ) according to KRAS and BRAF mutated versus non-mutated cancers. There is no significant association was observed between the level of NAIF1 expression for cancerous samples (T) according to KRAS and BRAF mutated versus nonmutated cancers.

Figure 3: NAIF1 expression according to comparison between 3 parameters where $\mathrm{P}=0.0001$. A: comparison between $(\mathrm{N}, \mathrm{T})$. B: comparison between (non-metastatic, metastatic). C: comparison between (MSI, MSS).

\section{Discussion}

Several studies have described the proteomic expression of NAIF1 gene ${ }^{[10-14]}$. Our results are consistent with the previous studies were done on NAIF1 gene expression showed that, NAIF1 is expressed in both cancer tissue or cancer cell lines and in non-cancerous or normal tissue ${ }^{[10,12]}$, this expression was strong or high in non-cancerous or normal tissue compared with weak or little seen in cancerous one. This finding may be interpreted by a possible relationship between gene expression and stage or grade of tumor (inversely related) and inturn possible role for this gene in the regression or inhibition in this type of cancer. Our results here in colorectal tissue cancerous and noncancerous are in coherence with Luo's finding in tissues, they demonstrated NAIF1 protein is highly expressed in human normal gastric tissue and down- regulated or lost in gastric cancer tissue ${ }^{[10]}$. These findings may indicate that it is very likely that NAIF1 may predominantly act as a tumor-suppressive gene, possibly through the activation of apoptotic pathways, in various types of cancers ${ }^{[10-14]}$. On the other hand, the high level of expression of NAIF1 in normal areas might suggest a role of NAIF1 in the control of genes with anti-tumor activity.

NAIF1 is significantly highly expressed in the tumor tissues of the colon with molecular phenotype of microsatellite instable (MSI) than in those of microsatellite stable (MSS) molecular phenotype. These results tend to suggest that NAIF1 could be associated with the mechanism of carcinogenesis of colorectal cancers of MSI molecular phenotype. Unfortunately, no information available in the bibliography tried to reveal this possible link between the genetic phenotype of cancer wether microsatellite instable (MSI) or microsatellite stable (MSS) status at the level of nucleotide and NAIF1 gene expression. To confirm the correlation, further research is required.

NAIF1 expression in the metastatic (low expression) versus non-metastatic (high expression) stage of colorectal cancer, is in coherence Luo's finding in tissues ${ }^{[10]}$. This may be assumed by either the gene has a role in inhibition the progression of cancer by inducing apoptosis or the highly progressed cancer express this gene little. Further investigation will be required to clarify this relationship.

\section{Conclusion}

The higher expression of NAIF1 protein in noncancerous colorectal tissues and lower expression in cancerous one, with higher expression in non-metastatic than metastatic one may indicates a relationship between cancer inhibition and regression with gene expression. There is a role of NAIF1 in colorectal carcinogenesis especially for cancers of microsatellite instable (MSI) phenotype. Therefore, NAIF1 may provide new clues for developing anti-cancer drugs.

Acknowledgments: We thank E. Goudeau (INSERM UMR 1087, Nantes) for her technical expertise in molecular biology, B. Pitard (Nantes university) for providing antibodies.

Source of Funding: This work was funded by the C.N.R.S, the I.N.R.A., the Groupement de Recherche CNRS 2157, and the Ministère de l'Education Nationale, de la Recherche et de la Technologie. It also received funding from a research program grant from the Cancéropôle Grand-Ouestand grants from Amgen and the French National Society of Gastroenterology.

Ethical Clearance: Samples were taken from the department of histopathology/Trousseau hospital/ Tours/France. Patients had been informed of using their samples for research purposes and their consents to participation in this type of research had been collected.

Conflict of Interest: There is no conflict.

\section{References}

1. Fearon ER, Vogelstein B. "A Genetic Model for Colorectal Tumorigenesis.” Cell. 1990;61:759767.

2. Chung DC. "The Genetic Basis of Colorectal 
Cancer: Insights into Critical Pathways of Tumorigenesis." Gastroenterol. 2000; 119:854865.

3. Boland C R, Goel A. "Somatic Evolution of Cancer Cells.” Semin Cancer Biol. 2005; 15:436-450.

4. Vogelstein B, Fearon ER, Kern SE, et al. "Allelotype of Colorectal Carcinomas." Science. 1989;244: 207-211.

5. Delattre O, Olschwang S, Law DJ, et al. "Multiple Genetic Alterations in Distal and Proximal Colorectal Cancer." Lancet. 1989;2: 353-356.

6. Sinzelle L, Izsvák Z, Ivics Z. "Molecular Domestication of Transposable Elements: From Detrimental Parasites to Useful Host Genes." Cell Mol Life Scis. 2009;66:1073-1093.

7. Feschotte C, Pritham EJ. "DNA Transposons and the Evolution of Eukaryotic Genomes." Annu Rev Genet. 2007;41:331-368.

8. Lander ES, Linton LM, Birren B, et al. "Initial Sequencing and Analysis of the Human Genome." Nature. 2001;409:860-921.

9. Sinzelle L, Kapitonov VV, Grzela DP, Jursch T, Jurka J, Izsvak Z, Ivics Z. Transposition of a reconstructed Harbinger element in human cells and functional homology with two transposon- derived cellular genes. Proc Natl Acad Sci U S A. 2008; 105:4715-4720.

10. Luo Q, Zhao M, Zhong J, et al. NAIF1 is downregulated in gastric cancer and promotes apoptosis through the caspase- 9 pathway in human MKN45 cells. Oncol Rep. 2011;25:1117-1123.

11. Yi Fu, Fuhua cao.Micro RNA-125a-5p regulates cancer cell proliferation and migration through naiF1 in prostate carcinoma. Onco Targets Ther. 2015;8:3827-35.

12. Yang $M, G u$ YY, Peng H, et al. NAIF1 inhibits gastric cancer cells migration and invasion via the MAPK pathways. J Cancer Res Clin Oncol. 2015;14:1037-1047.

13. Kong D, Zhang Z. NAIF1 suppresses osteosarcoma progression and is regulated by miR-128. Cell Biochem Funct. 2018;36(8):443-449.

14. Zhao G, Liu L, Zhao T, et al. Upregulation of miR-24 promotes cell proliferation by targeting NAIF1 in non-small cell lung cancer. Tumour Biol. 2015;36:3693-3701.

15. Arnaoty A, Pitard B, Bateau B, Bigot Y, Lecomte $T$ Novel Approach for the Development of New Antibodies Directed Against Transposase-Derived Proteins Encoded by Human Neogenes. Method Mol Biol. 2012;859:293-305. 